\title{
UM PANORAMA DO USO DE PESQUISA OPERACIONAL NO AMBIENTE DE GUERRA SUBMARINO
}

\author{
Leonardo Antonio Monteiro Pessôa \\ CASNAV \\ Departamento de Engenharia de Sistemas \\ lampessoa@terra.com.br \\ Edilson Fernandes de Arruda \\ COPPE/UFRJ \\ Programa de Engenharia de Produção \\ efarruda@po.coppe.ufrj.br \\ Laura Bahiense \\ COPPE/UFRJ \\ Programa de Engenharia de Sistemas e Computação \\ laura@cos.ufrj.br

\section{RESUMO}

Este artigo apresenta um panorama do uso de metodologias de pesquisa operacional aplicadas para a guerra em ambiente submarino. Apresenta uma revisão bibliográfica referente a base Scopus, no tocante ãs técnicas mais utilizadas, apresentando os países de origem, e agrupando os documentos para seu melhor entendimento. O trabalho contribui para um conhecimento mais profundo sobre de qual maneira as ferramentas de PO estão sendo utilizadas no domínio da guerra submarina, apresentando as tendências e desenvolvimentos mais recentes, podendo servir como uma orientação para futuras pesquisas brasileira neste tema.

Palavra-chave: Pesquisa Operacional; Defesa; Militar; Guerra A/S.

\begin{abstract}
This paper presents a panorama of applied use of Operations Research methodology on submarine warfare. It presents a bibliographic review based on Scopus database, exploring the most used techniques, clustering the documents and presenting the countries of origin for a better understanding. The paper provides a deeper knowledge about how OR has been used in this context, presenting the recent developments and tendencies, and may orient future Brazilian research of on this theme.
\end{abstract}

Keywords: Operations Research; Defense; Military; ASW.

Como Citar:

PESSÔA, Leonardo Antonio Monteiro; Arruda, Edilson Fernandes; BAHIENSE, Laura. 
Um panorama do uso de Pesquisa Operacional no ambiente de guerra submarino. In: SIMPÓSIO DE PESQUISA OPERACIONAL E LOGÍSTICA DA MARINHA, 19., 2019, Rio de Janeiro, RJ. Anais [...]. Rio de Janeiro: Centro de Análises de Sistemas Navais, 2019.

\section{INTRODUÇÃO}

O presente trabalho objetiva construir um panorama do uso de métodos de pesquisa operacional em aplicações de guerra no ambiente submarino. Para tanto, apresenta-se uma pesquisa bibliográfica com base na plataforma Scopus. A escolha dessa base bibliográfica é justificada pela sua grande abrangência, assim como sua aceitação junto à comunidade científica brasileira.

Cabe ressaltar que se trata de uma pesquisa ainda preliminar, em que pese o número significativo de referências encontradas. Pretende-se, portanto, enriquecer a pesquisa através da consulta a outas bases bibliográficas conhecidas, como Web of Science, ScienceDirect, entre outros.

Adicionalmente, é intencionada a captura de documentos que sejam originários de fontes de conhecimento indexadas, de maneira a possibilitar a identificação de tendências quanto à origem dos documentos. Pretende-se ainda inferir o comportamento temporal das publicações relativas ao tema, os tipos de publicação mais frequentes, além dos aspectos metodológicos mais explorados.

De modo a caracterizar metodologicamente a estrutura do trabalho, a próxima seção apresenta uma breve fundamentação teórica com o detalhamento da abordagem empregada na condução do presente trabalho. A seção 3 apresenta os resultados, com foco nas referências identificadas. Por fim, a seção 4 traz uma breve discussão dos resultados e a conclusão deste trabalho.

\section{FUNDAMENTAÇÃO TEÓRICA}

O interesse pelo ambiente de guerra antisubmarino está relacionada aos primórdios da Pesquisa Operacional organizada, especificamente a desenvolvimentos do "circo" de Blackett [1].

Por outro lado, vemos que há importância específica para o contexto brasileiro, denotada pela importância dada na Estratégia Nacional de Defesa [2] ao ambiente de guerra submarino.

Há menção [3] a referências neste contexto, principalmente teses e dissertações. No entanto, considera-se a Base Scopus como um repositório muito importante para a comunidade científica brasileira, incluindo periódicos de grande relevância, o que permite verificar os avanços metodológicos mais importantes e identificar tendências quanto a: temporalidade, origem e áreas de conhecimento envolvidas. Além de contribuir para identificar possibillidades de aplicação e de 
lacunas metodológicas.

Para este objetivo, detalha-se a seguir a metodologia de levantamento.

\subsection{METODOLOGIA DO LEVANTAMENTO}

Na fase inicial, o método utilizado para a condução da revisão bibliográfica consiste dos seguintes passos:

1. Pesquisa na base Scopus utilizando os termos "submarine" "warfare"e "Operations Research" ou "Operational Research"

2. Apresentação dos resultados relativo a:

Temporalidade

Origem

Áreas de Conhecimento

3. Mapeamento das palavras chave mais relevantes, utilizando o software vosviewer

4. Mapeamento das palavras mais relevantes constantes do abstract, utilizando o software vosviewer

5. Classificação dos itens quanto ao domínio e à Ferramenta de pesquisa operacional (PO) utilizada.

\section{RESULTADOS}

A tabela 1 mostra a entrada para consulta utilizada, a qual resultou inicialmente em 23 artigos.

\begin{tabular}{lll}
\hline Base & Query & Found Docs \\
\hline SCOPUS & TITLE-ABS-KEY ( ("submarine"AND "warfare") & 23 \\
& AND ( "Operations Research"OR "Operational Research") ) & \\
\hline
\end{tabular}

Tabela 1: Termos de pesquisa e resultados. Fonte: Autores

A figura 1 mostra o número de publicações levantadas por ano. Observa-se um comportamento irregular das publicações, com vários anos sem representação.

Todavia, nota-se o que parece ser o início de uma tendência de aumento de publicações a partir de 2016. Verifica-se, a partir daí, publicações todos os anos. Além disso, cabe observar que o maior número de publicações anuais dá-se exatamente no último ano, em 2018.

Isso sugere um aumento de interesse pelo tema nos últimos anos e o possível início de uma trajetória ascendente do número de publicações. 


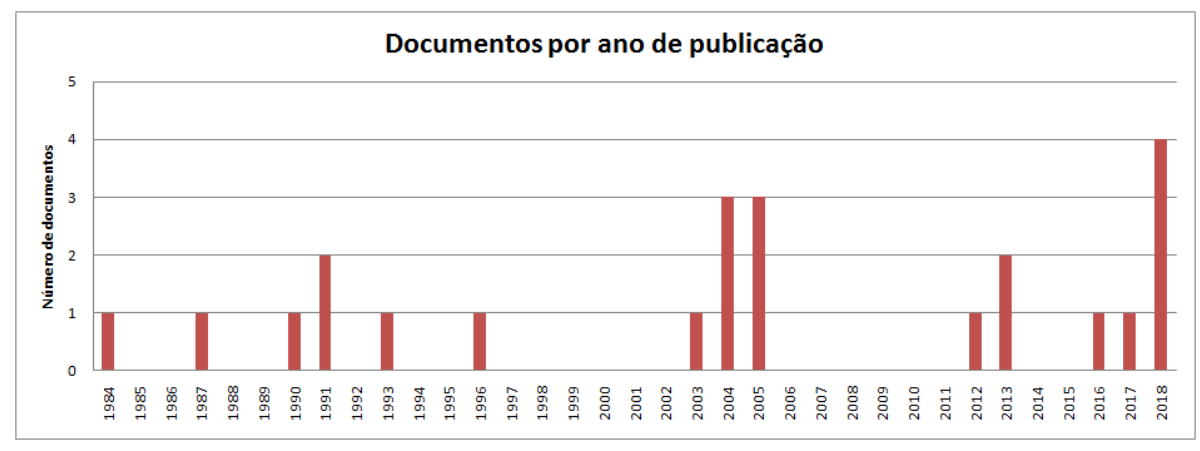

Figura 1: Documentos por ano. Fonte: Scopus (adaptado)
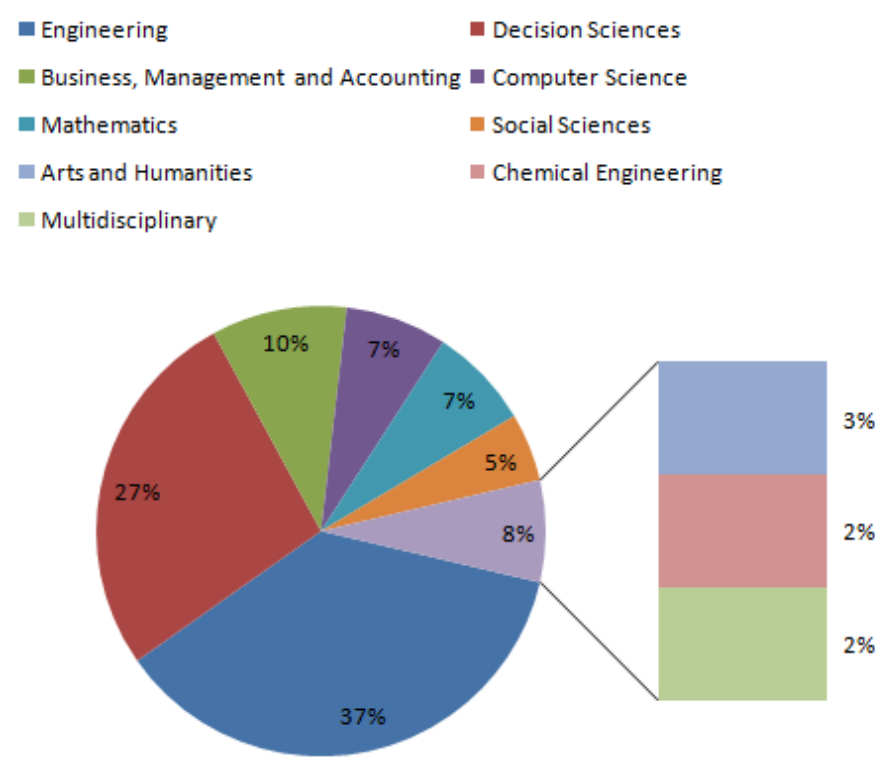

Figura 2: Documentos por área. Fonte: Scopus

Já a figura 2 coloca os artigos em perspectiva em função de sua área de conhecimento. Nota-se uma predominância de documentos afetos à área de engenharia e ciência da decisão, que representam mais de $60 \%$ dos documentos encontrados

A figura 3, por sua vez, evidencia uma predominância de literatura produzida nos Estados Unidos e na China. Não obstante haver indicação de seis entradas sem origem definida, ao analisar-se a afiliação dos autores, apresentada na figura 4. observa-se que a predominância mencionada é coerente.

A figura 5 apresenta um mapa das ocorrências das palavras-chave catalogadas, utilizando-se o software VOS viewer.

Para a construção deste mapa foi necessária a exclusão do documento [4], uma vez que foi detectado o corrompimento do arquivo ".csv" proveniente da Scopus, quando o mesmo era mantido.

Foi estabelecido o limite mínimo de três ocorrências para cada palavrachave. Com isso, restaram apenas as 14 palavras-chave mais importantes de um 


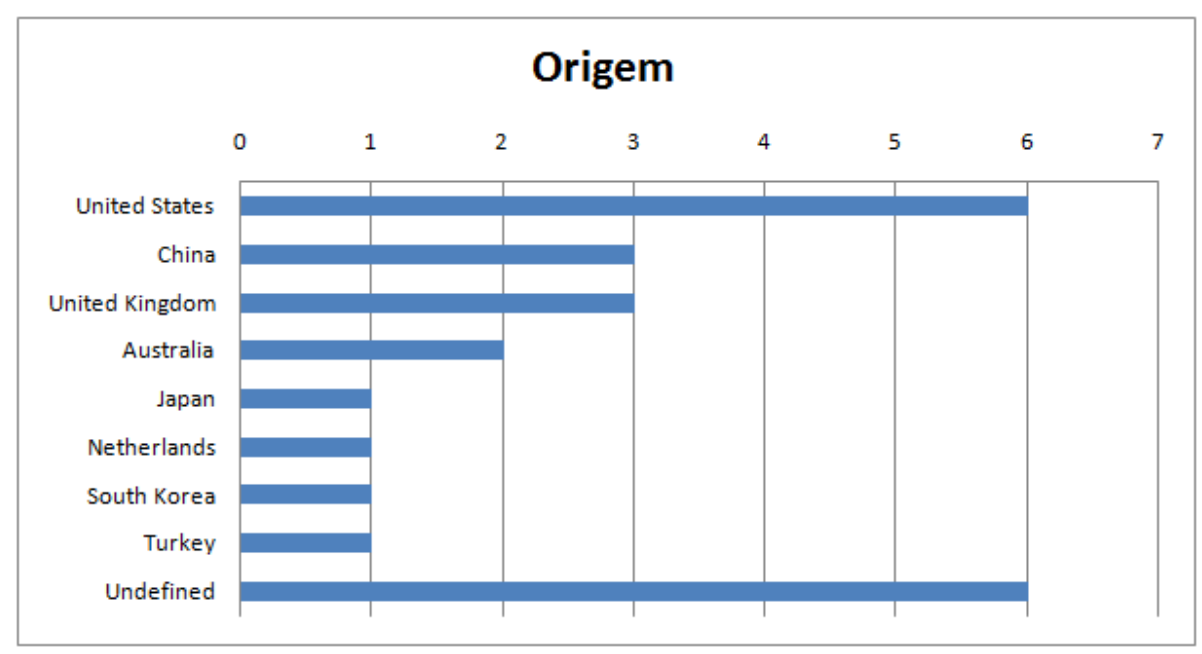

Figura 3: Documentos por país. Fonte: Scopus

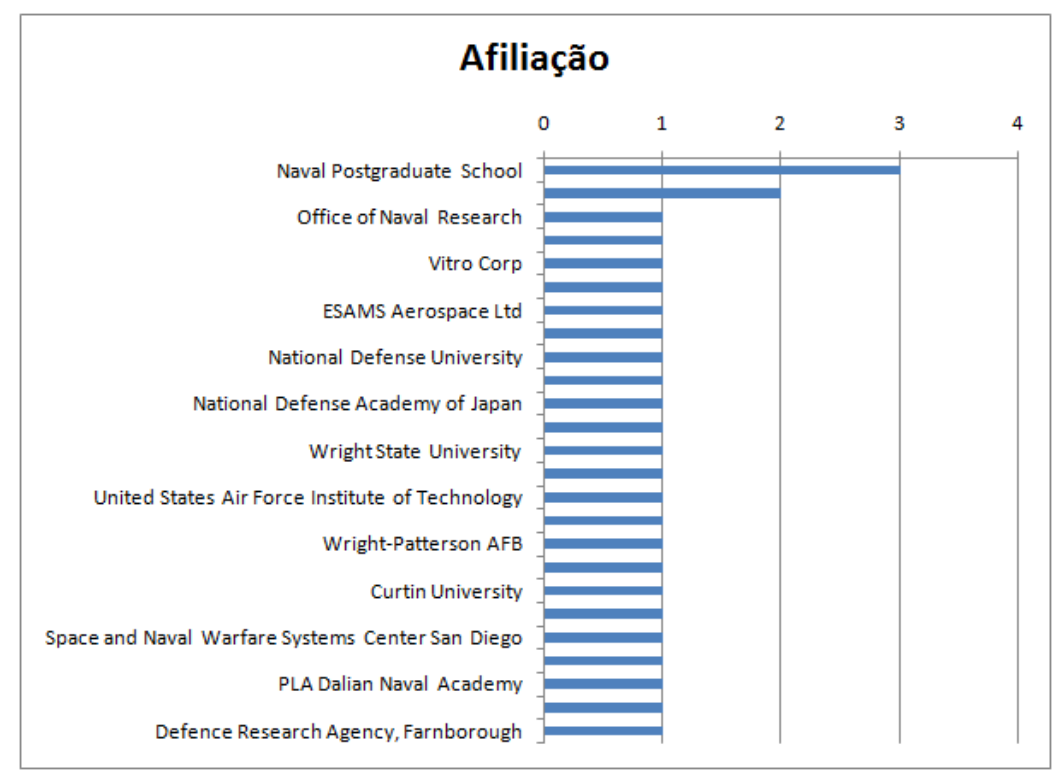

Figura 4: Documentos por afiliação. Fonte: Scopus

total de 175 .

Ainda na figura 5, nota-se a presença dos termos "anti-submarine warfare (asw)" e "anti-submarine warfares". Estes aparecem separadamente, mas em sendo unificados, possuiriam número de ocorrências igual ao do termo "submarines" (8 ocorrências).

A figura 6 traz uma perspectiva distinta. Nela, representa-se o mapa das mais frequents associações de palavras encontradas nos títulos e resumos (abstracts) dos artigos pesquisados. São marcantes as menções a "system"e "probability" que frequentemente têm papel de destaque na conexão entre os termos "asw", "antisubmarine warfare" e "target" aos termos "convoy" e "path".

Todavia a análise deste mapeamento não é suficiente para evidenciar os 


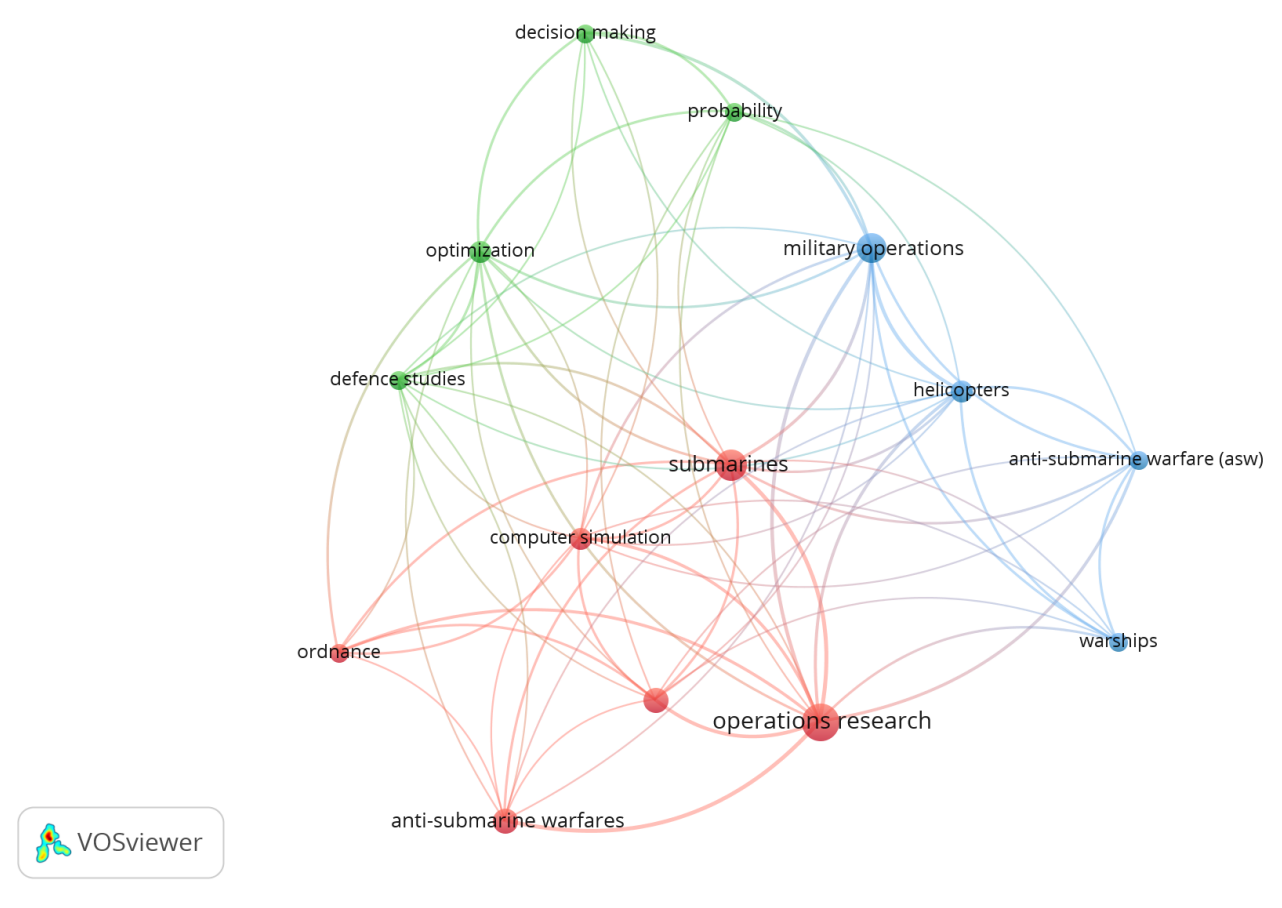

Figura 5: Keywords. Fonte: Autores

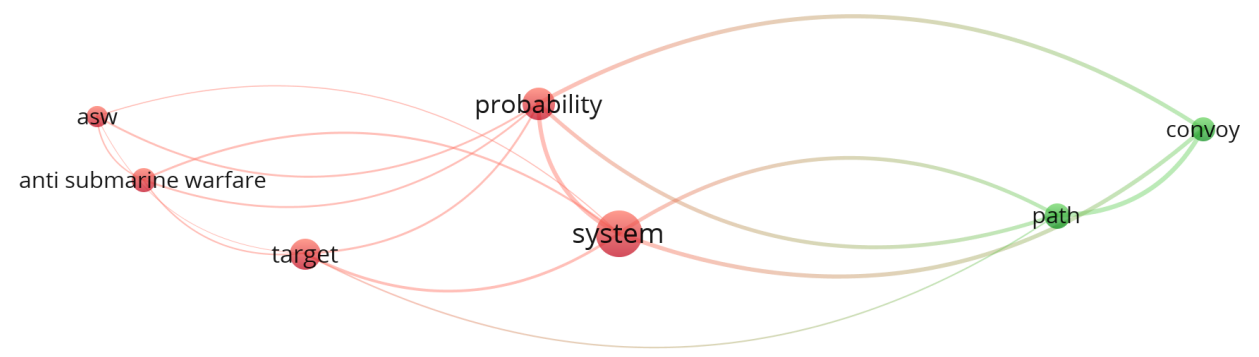

\& VOSviewer

Figura 6: Conexões mais frequentes Fonte: Autores

métodos de Pesquisa Operacional utilizados. Portanto, procedeu-se uma análise dos resumos para extração de mais informações a esse respeito, as quais são apresentadas na tabela 2. Verifica-se a repetição de um documento ([5]), de modo que se contam 22 documentos diferentes 


\begin{tabular}{|c|c|c|c|}
\hline Fonte & $\begin{array}{l}\text { Elementos } \\
\text { metodológicos } \\
\text { mencionados }\end{array}$ & Problema & Finalidade \\
\hline [4] & $\begin{array}{l}\text { integral geome- } \\
\text { try - geometric } \\
\text { probability - } \\
\text { Monte Carlo } \\
\text { Simulation }\end{array}$ & uso de sonobóias & $\begin{array}{l}\text { deteção de subma- } \\
\text { rino }\end{array}$ \\
\hline [6] & \begin{tabular}{lr}
\multicolumn{2}{l}{ nonzero-sum } \\
two-player \\
network inter- \\
diction & game \\
$-\quad$ Strong & Stac- \\
kelberg equili- \\
brium.
\end{tabular} & $\begin{array}{l}\text { alocação de } \quad \text { recursos- } \\
\text { Patrulha em "chokepoints" }\end{array}$ & $\begin{array}{l}\text { oposição ao subma- } \\
\text { rino }\end{array}$ \\
\hline [7] & search pattern & 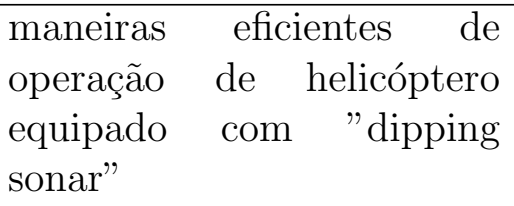 & $\begin{array}{l}\text { deteção de subma- } \\
\text { rino }\end{array}$ \\
\hline [8] & simulação & experimentação sequencial & $\begin{array}{l}\text { engajamento de } \\
\text { combate }\end{array}$ \\
\hline [9] & $\begin{array}{l}\text { comparação } \\
\text { histórica }\end{array}$ & $\begin{array}{l}\text { exame do impacto de medi- } \\
\text { das e contramedidas na asw }\end{array}$ & lições históricas \\
\hline 10 & search theory & search game & $\begin{array}{l}\text { revisão } \quad \text { bibli- } \\
\text { ográfica }\end{array}$ \\
\hline 5 & $\begin{array}{l}\text { information en- } \\
\text { tropy }\end{array}$ & $\begin{array}{l}\text { operação ASW por navio de } \\
\text { superfície }\end{array}$ & $\begin{array}{l}\text { medição de eficácia } \\
\text { - avaliação da quali- } \\
\text { dade de informação }\end{array}$ \\
\hline [11] & $\begin{array}{l}\text { modelo ma- } \\
\text { temático }\end{array}$ & $\begin{array}{l}\text { cálculo de ângulo de ori- } \\
\text { entação dos torpedos em } \\
\text { relação ao alvo - torpedos de } \\
\text { busca acústica }\end{array}$ & $\begin{array}{l}\text { engajamento de } \\
\text { combate }\end{array}$ \\
\hline 12 & $\begin{array}{l}\text { otimização dis- } \\
\text { creta }\end{array}$ & shortest path problem & $\begin{array}{l}\text { medição de eficácia } \\
\text { de sistemas ISR - } \\
\text { proteção de com- } \\
\text { boio }\end{array}$ \\
\hline 13 & - & $\begin{array}{l}\text { supervisão de unidades } \\
\text { navais em alta demanda } \\
\text { durante operações de } \\
\text { baixa intensidade desen- } \\
\text { volvimento de tecnologias } \\
\text { para condução de "anti- } \\
\text { submarine warfare" }\end{array}$ & $\begin{array}{l}\text { discussão / notícia } \\
\text { de defesa }\end{array}$ \\
\hline
\end{tabular}




\begin{tabular}{|c|c|c|c|}
\hline 14 & search theory & $\begin{array}{l}\text { two-sided, multi-stage se- } \\
\text { arch problem busca de sub- } \\
\text { marino inimigo por força } \\
\text { naval }\end{array}$ & $\begin{array}{l}\text { deteção de subma- } \\
\text { rino }\end{array}$ \\
\hline 15 & - & $\begin{array}{lll}\text { discussão sobre armas de } \\
\text { destruição em massa }\end{array}$ & $\begin{array}{l}\text { discussão / notícia } \\
\text { de defesa }\end{array}$ \\
\hline 16 & - & 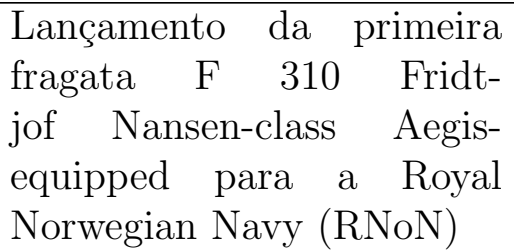 & $\begin{array}{l}\text { discussão / notícia } \\
\text { de defesa }\end{array}$ \\
\hline [17] & $\begin{array}{l}\text { modelo ma- } \\
\text { temático }\end{array}$ & $\begin{array}{l}\text { Comprehensive } \text { Maritime } \\
\text { Patrol Aircraft Systems } \\
\text { Simulation (COMPASS) }\end{array}$ & $\begin{array}{l}\text { eficácia operacional } \\
\text { (predição) }\end{array}$ \\
\hline 18 & $\begin{array}{l}\text { search theory, } \\
\text { agent-based } \\
\text { simulation }\end{array}$ & $\begin{array}{l}\text { estudo de caso histórico en- } \\
\text { volvendo busca ofensiva }\end{array}$ & $\begin{array}{l}\text { deteção de subma- } \\
\text { rino - engajamento } \\
\text { em combate }\end{array}$ \\
\hline 19 & $\begin{array}{l}\text { oceanografia, } \\
\text { acústica, e PO }\end{array}$ & $\begin{array}{l}\text { support tactical decision } \\
\text { making in military missions }\end{array}$ & $\begin{array}{l}\text { deteção - engaja- } \\
\text { mento em combate }\end{array}$ \\
\hline 20 & simulação & $\begin{array}{l}\text { método e ferramentas de si- } \\
\text { mulação para design e de- } \\
\text { senvolvimento }\end{array}$ & $\begin{array}{l}\text { desenvolvimento de } \\
\text { sistemas de guerra } \\
\text { abaixo d'água para } \\
\text { navios de superfície }\end{array}$ \\
\hline 21 & probabilidade & $\begin{array}{l}\text { nova abordagem para quan- } \\
\text { tificar a probabilidade de } \\
\text { sucesso de missão ASW sem } \\
\text { destruir submarino }\end{array}$ & $\begin{array}{l}\text { mensuração de } \\
\text { eficácia }\end{array}$ \\
\hline 22 & - & $\begin{array}{l}\text { apresentar assessoramento } \\
\text { técnico e científico para } \\
\text { SACLANT (Supreme Al- } \\
\text { lied Commander Atlantic) e } \\
\text { nações da OTAN no campo } \\
\text { de guerra antissubmarino }\end{array}$ & $\begin{array}{l}\text { discussão / notícia } \\
\text { de defesa }\end{array}$ \\
\hline 23 & - & $\begin{array}{l}\text { ataque de torpedo subma- } \\
\text { rino, especificamente enga- } \\
\text { jamentos de salvas contra } \\
\text { múltiplos alvos }\end{array}$ & $\begin{array}{l}\text { engajamento } \\
\text { combate }\end{array}$ \\
\hline 24 & $\begin{array}{l}\text { computer simu- } \\
\text { lation }\end{array}$ & $\begin{array}{l}\text { representar situações táticas } \\
\text { envolvendo dois submarinos } \\
\text { oponentes }\end{array}$ & $\begin{array}{l}\text { engajamento de } \\
\text { combate }\end{array}$ \\
\hline 25 & Markov process & $\begin{array}{l}\text { maximização da probabili- } \\
\text { dade de deteção de um sub- } \\
\text { marino }\end{array}$ & $\begin{array}{l}\text { deteção de um sub- } \\
\text { marino }\end{array}$ \\
\hline
\end{tabular}

Tabela 2: Referências Encontradas. Fonte: Scopus 
Nota-se preponderância das finalidades relacionadas a: deteção, engajamento de combate e mensuração de eficácia. Mesmo nos documentos voltados para aspectos históricos, a análise compreende o aperfeiçoamento da tática através do método científico.

\section{DISCUSSÃO E CONCLUSÃO}

A literatura encontrada, apesar de irregular no tempo, mostra interesse renovado nos últimos anos. É um campo com desenvolvimento mais centrado nos Estados Unidos e China, e em instituições ligadas às Marinhas.

Percebe-se nos documentos a concentração em área de conhecimento voltada aos métodos de engenharia, matemática e tomada de decisão, devotadas para aplicação direta na guerra antissubmarino, a qual tem por objetivo encontrar, perseguir e deter, avariar ou destruir submarinos inimigos. Vê-se como ramo mais robusto de dicussão o tópico de detecção de submarino e de busca. São também destacados aspectos de engajamento de combate e de mensuração de eficácia.

Nesse universo de Guerra no ambiente submarino pode ser notada uma razoável diversidade nos métodos empregados, desde modelos de otimização e heurísticas, passando por análise matemática, simulação, e teoria de jogos. Esta diversidade mostra que os problemas encontrados podem ser encarados sob diversas abordagens.

Reconhece-se que também está presente uma significante parcela não devotada à Pesquisa Operacional em si, na qual enconram-se notícias e discussões sobre defesa. Estas contribuições servem como elemento para contextualização do interesse político-militar no tema, ajudando na construção do panorama.

A existência de publicações relacionadas ao tema publicadas em veículos importantes de PO demonstra o interesse do tema para a literatura e, dada a aproximação da Marinha às instituições federais de ensino e pesquisa, mostra-se interessante o desenvolvimento de pesquisa nacional nesse tema específico. 


\section{REFERÊNCIAS BIBLIOGRÁFICAS}

[1] TREFETHEN, F. N. A history of Operations Research. In: Fundamentals of Naval Operational Analysis. Annapolis: United States Naval Institute, 1970. cap. A history, p. 188-205. 1

[2] BRASIL. Estratégia Nacional de Defesa. 2012. 1

[3] PESSôA, L. A. M. et al. Pesquisa Operacional na Marinha Do Brasil : O Casnav , seu presente e perspectivas. In: SBPO. Vitória: [s.n.], 2016. p. 2302-2312. 1

[4] KARATAS, M.; CRAPARO, E.; AKMAN, G. Bistatic sonobuoy deployment strategies for detecting stationary and mobile underwater targets. Naval Research Logistics (NRL), v. 65, n. 4, p. 331-346, jun. 2018. ISSN 0894069X. 3. 6

[5] LIU, J.; XUE, C.-Y.; WANG, Y.-J. A method of information quality assessment of ship ASW operation. In: 11th International Symposium on Operations Research and its Applications in Engineering, Technology and Management 2013, ISORA 2013. Academy of Navy Submarine, Qingdao 266071, China: Institution of Engineering and Technology, 2013. p. 1-4. ISBN 9781849197137 (ISBN). 5, 6

[6] HEW, P.; YIAP, N. Optimally randomized patrolling of chokepoints for theatre antisubmarine warfare. Military Operations Research, Military Operations Research Society, Department of Defence, Australia, v. 23, n. 1, p. 49-56, 2018. ISSN 02755823 (ISSN). 6

[7] YOASH, R. B.; ATKINSON, M. P.; KRESS, M. Where to dip? Search pattern for an antisubmarine helicopter using a dipping sensor. Military Operations Research, Military Operations Research Society, Naval Postgraduate School, United States, v. 23, n. 2, p. 19-39, 2018. ISSN 02755823 (ISSN). 6

[8] KIM, J. H. et al. Achieving new insights into combat engagement analysis via simulation-based sequential experimentation. Military Operations Research, Military Operations Research Society, Agency for Defense Development, South Korea, v. 23, n. 4, p. 51-80, 2018. ISSN 02755823 (ISSN). 6

[9] DUFFEY, R. B. Submarine warfare and intelligence in the Atlantic and Pacific in the Second World War: comparisons and lessons learned for two opponents. Journal for Maritime Research, v. 19, n. 2, p. 143-167, jul. 2017. ISSN 2153-3369. 6

[10] HOHZAKI, R. Search games: Literature and survey. Journal of the Operations Research Society of Japan, v. 59, n. 1, p. 1-34, 2016. ISSN 0453-4514. 6

[11] XIA, Z.-J.; ZHANG, X.-H.; XU, L.-Z. Study on orientation angle of two acoustic homing torpedoes relative to target with parallel salvo launched 
by submarine. Binggong Xuebao/Acta Armamentarii, Department of Information and Communication Engineering, Dalian Navy Academy, Dalian 116018, Liaoning, China, v. 33, n. 5, p. 636-640, 2012. ISSN 10001093 (ISSN). Disponível em: <https://www.scopus.com/inward/record.uri?eid=2s2.0-84863567715\&partnerID $=40 \& m d 5=b 87 d 3 c 46 c c 5 d 946 c 31 c 9 f 8 b 11 d 5710 e a>$. 6

[12] GLASSBOROW, D. et al. Estimating the value of intelligence, surveillance and reconnaissance in Manoeuvre Warfare. In: MODSIM05 - International Congress on Modelling and Simulation: Advances and Applications for Management and Decision Making. Maritime Operations Division, Defence Science and Technology Organisation, Building A51, Rockingham, WA 6958, Australia: [s.n.], 2005. p. 1758-1764. ISBN 0975840002 (ISBN); 9780975840009 (ISBN). Disponível em: <https://www.scopus.com/inward/record.uri?eid=2s2.0-80053123909\&partnerID $=40 \& m d 5=b 53 e 545399 \mathrm{aec} 0 \mathrm{ae} 2422 \mathrm{e} 0840 \mathrm{f0653c4}>$. 6

[13] KOCH, A. US Navy outlines expeditionary command plans. Jane's Defence Weekly, n. OCT., 2005. ISSN 02653818 (ISSN). Disponível em: $\quad<$ https://www.scopus.com/inward/record.uri?eid=2-s2.0$84861270651 \&$ partnerID $=40 \& m d 5=c 412906 \mathrm{e} 23 \mathrm{aa} 8280 \mathrm{a} 71 \mathrm{aba} 66086 \mathrm{~b} 32 \mathrm{c} 0>$. 6

[14] VERMEUlEN, J. F. J.; Van Den Brink, M. The search for an alerted moving target. Journal of the Operational Research Society, TNO Phys. and Electronics Laboratory, The Hague, Netherlands, v. 56, n. 5, p. 514-525, 2005. ISSN 01605682 (ISSN). 7

[15] Schneider Jr., W. A 21st-century role for nuclear weapons. Issues in Science and Technology, Defense Science Board, Department of Defense, v. 20, n. 3, p. 55-60, 2004. ISSN 07485492 (ISSN). Disponível em: <https://www.scopus.com/inward/record.uri?eid=2-s2.02442645102\&partnerID=40\&md5=1491e0948b739a587b9dec1a34890341>. 7

[16] LOK, J. J. Norway's Fridtjof Nansen-class frigates to provide a key strategic advantage. Jane's International Defence Review, n. MAR., 2004. ISSN 14762129 (ISSN). Disponível em: <https://www.scopus.com/inward/record.uri?eid=2s2.0-50049123095\&partnerID=40\&md5=af0bccfb847d0c154ea47775ab06bf0e $>$. 7

[17] BRENNAN, J. F. M.; DENTON, A. L. Compass - the verification and validation of an operational analysis model for use in the prediction of Nimrod MRA4 operational effectiveness. Journal of the Operational Research Society, Integrated Defence Analysis Team, BAE SYSTEMS, Warton, Preston, Lancashire, United Kingdom, v. 55, n. 4, p. 413-421, 2004. ISSN 01605682 (ISSN). 7

[18] CHAMPAGNE, L.; Greg Carl, R.; HILL, R. Search theory, agent-based simulation, and U-boats in the bay of biscay. In: S.E., C. et al. (Ed.). 2003 Winter 
Simulation Conference: Driving Innovation. Department of Operational Sciences, Air Force Institute of Technology, Wright Patterson AFB, OH 45433, United States: [s.n.], 2003. v. 1, p. 991-998. ISBN 02750708 (ISSN). 7

[19] INCZE, B. I. Tactical oceanographic battlespace. Sea Technology, Compass Publications Inc, Arlington, VA, United States, v. 37, n. 8, p. 43-49, 1996. ISSN 00933651 (ISSN). 7

[20] NEVEU, D.; PIGNON, J.-P. Simulation for underwater warfare systems design . Revue technique - Thomson-CSF, THOMSON SINTRA, v. 25, n. 2, p. 669-682, 1993. ISSN 00354279 (ISSN). Disponível em: <https://www.scopus.com/inward/record.uri?eid=2-s2.00027617637\&partnerID=40\&md5=562a405c1b784f191e370fd2b47989a5> 7

[21] CRAWFORD, J. D.; SAWYER, F. L. ASW measures of effectiveness. Vitro Technical Journal, Vitro Corp, Norfolk, United States, v. 9, n. 1, p. 12-19, 1991. ISSN 1054481X (ISSN). 7

[22] MARTIN, R. L. SACLANTCEN: submarine threat neutralizer. Sea Technology, Office of Naval Research, London, United Kingdom, v. 32, n. 5, 1991. ISSN 00933651 (ISSN). Disponível em: <https://www.scopus.com/inward/record.uri?eid=2-s2.00026155264\&partnerID =40\&md5=06ec73155d6dad63116a5938fe2d8a85> . 7

[23] URBAN, C. D. Design and evaluation of a tactical decision aid. In: 1990 IEEE International Conference on Systems, Man, and Cybernetics. US Naval Ocean Syst Center, San, Diego, CA, USA: Publ by IEEE, 1990. p. 812-814. ISBN 08843627 (ISSN); 0879425970 (ISBN). 7

[24] KNAPP, B. M.; DUDLEY, A. R.; RYDER, J. S. Modelling techniques for simulation of submarine engagements. Journal of the Operational Research Society, ESAMS Ltd., Camberley, Surrey, United Kingdom, v. 38, n. 10, p. 891-898, 1987. ISSN 01605682 (ISSN). 7

[25] EAGLE, J. N. Optimal search for a moving target when the search path is constrained. Operations Research, US Naval Postgraduate Sch, Monterey,, CA, USA, US Naval Postgraduate Sch, Monterey, CA, USA, v. 32, n. 5, p. 1107-1115, 1984. ISSN 0030364X (ISSN). 7 